\title{
artigo
}

Leite, A. C. F., Giovanardi, J. M. L., Silva, F. M. R., Quadros, K. A. N, Santos, R. C., Andrade, S. N.

Preparo dos profissionais de enfermagem no atendimento a mulheres vítimas de violência sexual

\section{Preparo dos profissionais de enfermagem no atendimento a mulheres vítimas de violência sexual}

\author{
Preparing nursing professionals to care for women victims of sexual violence \\ Preparación de profesionales de enfermería para asistir a mujeres víctimas de violencia sexual
}

\section{RESUMO}

Objetivo: Identificar se os profissionais de enfermagem estão aptos para acolher as vítimas de violência sexual de forma humanizada. Métodos: A pesquisa se trata de revisão sistemática da literatura, realizada nos meses de setembro/2019 a agosto/2020. A coleta de dados se deu por meio de pesquisa nas bases de dados LILACS e BDENF. Como dificuldade para o estudo, encontramos a baixa quantidade de artigos sobre assunto abordado. Resultados: Evidenciou-se que os profissionais de enfermagem não estão preparados para o acolhimento de vítimas de violência sexual, em dois estudos mostrou o despreparo por fatores psicológicos, insegurança, em três estudos foi apontado falta de capacitação. Conclusão: Apesar de todos os protocolos, preparo teórico, prático e embasamento científico ao longo da formação acadêmica do profissional de saúde, o atendimento de enfermagem ainda é falho. É preciso haver uma equipe multidisciplinar alinhada e capacitada para acolher e dar continuidade ao atendimento humanizado às mulheres vítimas de violência sexual.

DESCRITORES: Cuidados de Enfermagem; Acolhimento; Violência Sexual; Delitos Sexuais; Enfermagem.

\section{ABSTRACT}

Objective: The aim of this study was to verify whether nursing professionals are apt to admit women that are victims of sexual violence following humanization guidelines. Methods: To do that, a systematic review of the theoretical framework was done through LILACS and BDENF databases from September 2019 to August 2020. This way, data was collected for the analysis although it was also verified the existence of a low number of papers on the subject. This reinforces the relevance of the present study to the area. Results: The results of the analysis show that the nursing professionals are not prepared to provide an appropriate service to women that are victims of sexual violence due to psychological factors, lack of preparation and training. In two studies showed unpreparedness by psychological factors, insecurity, in three studies a lack of training was pointed out. Conclusion: Despite all the protocols, the theoretical and practical background, the scientific support throughout the academic training of health professionals, nursing care is still flawed. It is the conclusion of this study that it is necessary to have a multidisciplinary team aligned and trained to admit and follow-up women that are victims of sexual violence according to the humanization guidelines.

DESCRIPTORS: Admittance; Nursing Care; Sexual Offenses; Sexual Violence; nursing

\section{RESUMEN}

Objetivo: El objetivo de este estudio es identificar si los profesionales de enfermería son capacitados para acoger a las víctimas de violencia sexual de manera humanizada. Métodos: Para eso, se utilizó como recurso metodológico la revisión sistemática de la literatura, realizada entre el periodo de septiembre/2019 hasta agosto/2020. La recolección de datos se llevó a cabo a través de la investigación en las bases de datos LILACS, BDENF, el estudio encontró dificultades debido al escaso número de artículos sobre el tema abarcado. Resultados: Los resultados demostraron que los profesionales de enfermería no están preparados para recibir víctimas de violencia sexual, debido a factores psicológicos, falta de preparo o falta de formación. En dos estudios se evidenció falta de preparación por factores psicológicos, inseguridad, en tres estudios se señaló falta de formación.

Conclusión: Se puede concluir que, pese a todos los protocolos, preparación teórica, práctica y base científica, a lo largo de la formación académica del profesional de la salud, la atención de enfermería es todavía deficiente. La investigación encuentra que es necesario que exista un equipo multidisciplinario alineado y capacitado para acoger de manera continuada la atención humanizada a las mujeres víctimas de violencia sexual.

DESCRIPTORES: Atención en Enfermería; Acogida; Violencia Sexual; Delitos Sexuales; enfermeira

RECEBIDO EM: 15/07/2021 APROVADO EM: 10/08/2021 


\section{ANA CAROLINA FERREIRA LEITE}

Graduanda em Enfermagem, Universidade do Estado de Minas Gerais, Minas Gerais, Brasil.

ORCID: 0000-0003-3635-4161

\section{JÚLIA MARIA LOPES GIOVANARDI}

Graduada em Enfermagem, Universidade do Estado de Minas Gerais, Minas Gerais, Brasil.

ORCID: 0000-0002-1633-7973

\section{FERNANDA MARCELINO DE REZENDE E SILVA}

Mestre em Enfermagem pela UFMG, Docente e Coordenadora do Curso de Graduação em Enfermagem da Universidade do Estado de Minas Gerais. Docente do Curso de Graduação em Enfermagem da Universidade de Itaúna (UIT).

ORCID: 0000-0003-2236-7009

\section{KARLA AMARAL NOGUEIRA QUADROS}

Enfermeira. Mestre em Educação, Cultura e Organização Social pela Universidade do Estado de Minas Gerais. Minas Gerais, Brasil. Docente do Curso de Graduação em Enfermagem da Universidade do Estado de Minas Gerais (UEMG), Unidade Divinópolis.

ORCID: 0000-0002-3750-4873

\section{REGINA CONSOLAÇÃO DOS SANTOS}

Enfermeira. Mestre em Ciência pela Universidade Federal de São João Del Rei, Campus Centro Oeste Dona Lindu UFSJ - (CCO). Docente do Curso de Graduação em Enfermagem da Universidade do Estado de Minas Gerais (UEMG), Unidade Divinópolis. ORCID: 0000-0002-7393-3210

\section{SILMARA NUNES ANDRADE}

Enfermeira. Doutora em Ciências da Saúde pela Universidade Federal de São João Del Rei, Campus Centro Oeste Dona Lindu UFSJ - (CCO). Docente do Curso de Graduação em Enfermagem da Universidade do Estado de Minas Gerais (UEMG), Unidade Divinópolis.

ORCID: 0000-0002-1975-0827

\section{INTRODUÇÃO}

A violência sexual é definida a partir das múltiplas formas de qualquer tentativa de obter um ato sexual forçado, comentários sexuais indesejados $\mathrm{e}$ atos direcionados ao tráfico sexual. No Brasil, define-se estupro como: "constranger alguém, mediante violência ou grave ameaça, a ter conjunção carnal ou a praticar ou permitir que com ele se pratique outro ato libidinoso"(1-2).

Qualquer pessoa está sujeita a sofrer algum tipo de violência sexual, em algum momento da vida, independente do sexo, classe social, cor, idade. Apesar disso, as mulheres jovens são a classe que mais sofre esse tipo de violência, além de sofrer agressōes(2).

Em uma pesquisa realizada pela Organização das Naçōes Unidas (ONU) no mundo, uma em cada cinco mulheres será vítima de estupro ou de tentativa. A violência contra a mulher é uma questão de saúde pública, exigindo que os estabelecimentos responsáveis por essa área sejam eficientes e eficazes. Muitos dos estupros não são notificados às autoridades, e os motivos disto são diversos, como a humilhação, medo da reação do parceiro(3).

A violência sexual traz inúmeros prejuízos para as vítimas, tanto a curto quanto a longo prazo, como uma gravidez indesejada, uma contaminação por uma infecção sexualmente transmissível (IST). A longo prazo, pode causar distúrbios sexuais às mulheres. Vítimas de violência sexual têm uma maior predisposição a sintomas psiquiátricos, depressão, síndrome do pânico(2).

Com relação ao atendimento das mulheres que passaram por violência, este deve ser realizado em um local adequado, de preferência em um local de maior privacidade possível para a realização da triagem e da entrevista. Para que possa ocorrer um atendimento de qualidade, é necessário que se estabeleça uma relação de confiança com a cliente(4).
O acolhimento de uma pessoa vítima de violência sexual deve se fazer em ambiente seguro, porém se diferencia da triagem. Esse acolhimento é muito importante, uma vez que é a partir dele que haverá o estabelecimento de um vínculo entre paciente e profissional de saúde. Deve ser realizado com sigilo, privacidade e ética. A pessoa deve ser atendida, de forma humanizada, em um local onde sua privacidade seja garantida, e, nesse momento, é preciso observar se há alguém junto à cliente que possa ser ligado ao crime(5).

Nos serviços de saúde, a mulher violentada sexualmente necessita de acolhimento, fator fundamental para a humanização da assistência à saúde e essencial para que se estabeleça um relacionamento de forma adequada entre o profissional e a cliente(6).

As unidades hospitalares do Sistema Único de Saúde (SUS) estão se capacitando para realizar a coleta de informaçōes e de vestígios em vítimas de violência sexual. $\mathrm{O}$ Ministério da Saúde publicou portaria que 


\section{artigo}

Leite, A. C. F., Giovanardi, J. M. L., Silva, F. M. R., Quadros, K. A. N., Santos, R. C., Andrade, S. N.

Preparo dos profissionais de enfermagem no atendimento a mulheres vítimas de violência sexual

explana o que fazer para atender uma vítima de violência sexual. As unidades que forem para isso qualificadas serão capazes de realizar o registro de informações em uma ficha de atendimento multiprofissional, como a coleta de material para uma análise futura por legistas(7).

Os exames devem ser realizados em estabelecimentos hospitalares classificados como Serviços de Referência para Atenção Integral às Pessoas em Situação de Violência Sexual, mediante o apoio de uma equipe composta por profissionais de diversas áreas, para prestar o melhor atendimento possível à vítima de violência sexual(7).

$\mathrm{O}$ primeiro contato estabelecido entre o profissional e a vítima deve ter um clima afetuoso, que não julgue a situação. É de extrema importância que os profissionais avaliem sua postura e seu emocional para lidar com o caso e discutir sobre a situação. Entre os profissionais que irão prestar esse primeiro cuidado estarão o enfermeiro e a equipe de enfermagem(8).

A enfermagem, como área que visa ao cuidar, tem de aprofundar-se em como se pratica o cuidar. Numa população que está nas mãos da enfermagem para ser acolhida e receber cuidados típicos de vítimas de violências sexuais ou abusos sexuais, a mulher como a principal atingida por essa situação ganha maior destaque, demandando conhecimento e cuidado humanizado e holístico. O primeiro contato que a vítima tem com o serviço de saúde é por parte da enfermagem, que irá oferecer-lhe um atendimento humanizado, anamnese detalhada, coleta de material para exames a serem realizados, administração de medicamentos e agendamento para futuros retornos(9).

Este estudo destina-se a compreender como os profissionais de enfermagem lidam com o atendimento a mulheres vítimas de violência sexual. Diante desse exposto, pergunta-se: os profissionais de enfermagem estão preparados para acolher e realizar um atendimento humanizado às vítimas de violência sexual?

\section{MÉTODOS}

Trata-se de uma revisão sistemática da

literatura que usou como recomendação os Principais Itens para Relatar Revisões Sistemáticas e Meta-análises (PRISMA) como instrumento. A pergunta de pesquisa foi baseada no acrônimo PICO que representa a População/Paciente, Intervenção, Intervenção de Comparação e "Outcomes”. Para a seleção das publicações. Para o levantamento nas bases citadas, foram utilizados os descritores em Ciência da Saúde (DeCS): delitos sexuais e enfermagem. Para as combinações, foi utilizado o operador booleano "AND".

Os critérios de inclusão foram artigos realizados no Brasil, sem delimitação de data de publicação, os quais responderam à pergunta norteadora "Os profissionais de enfermagem estão preparados para atender mulheres vítimas de violência sexual?". Os critérios de exclusão foram artigos dupli-

\section{Figura 1 - Fluxograma de informação da revisão sistemática, Divinópolis, Minas Gerais, 2020}

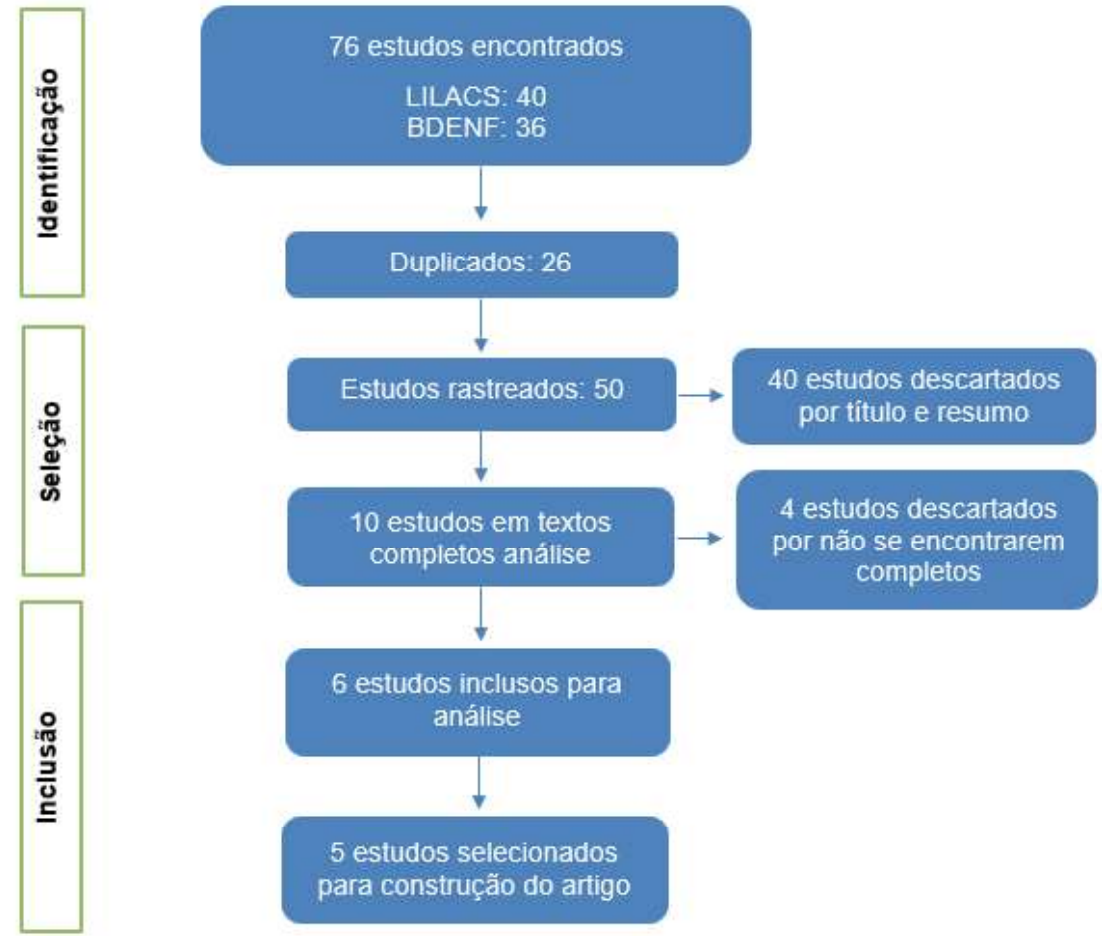

Fonte: Elaborada pelos autores, 2020

cados que não se apresentavam completos e que não abrangiam o tema da questão norteadora.

As bases de dados consultadas foram $\mathrm{Li}$ teratura Latino-Americana em Ciências de
Saúde (LILACS) e Base de Dados Bibliográficos Especializada na Área de Enfermagem (BDENF).

A busca nas bases de dados foi realizada de setembro/2019 a agosto/2020, no qual tivemos como resultados: 43 estudos na LILACS e 36 estudos na BDENF. Posteriormente foi realizada a leitura dos títulos e resumos de cada artigo buscando identificar quais respondiam à pergunta norteadora. Na LILACS, foram excluídos 8 por não apresentarem texto completos, 28 foram excluídos por não se encaixarem no tema e 4 selecionados. Já na BDENF foram excluídos 3 por não apresentarem textos completos 28 não se encaixavam no tema, 4 se adequavam ao tema, desses 3 estavam duplicados e foram excluídos. Quando os textos foram cruzados, foi encontrado 26 artigos duplicados (Figura 1). 
Quadro 1 - Estudos elegiveis mediante as bases de dados LILACS e BDENF, Divinópolis, Minas Gerais, 2020.

\begin{tabular}{|c|c|c|}
\hline TÍTULO & $\begin{array}{c}\text { OBJETIVO/ } \\
\text { METODOLOGIA }\end{array}$ & RESULTADOS \\
\hline $\begin{array}{l}\text { A percepção da vítima de } \\
\text { violência sexual quanto ao } \\
\text { acolhimento em um hospital } \\
\text { de referência no Paraná(10) }\end{array}$ & $\begin{array}{c}\text { Identificar a percepção } \\
\text { das vítimas de violência } \\
\text { sexual em relação ao } \\
\text { acolhimento prestado } \\
\text { pela equipe de } \\
\text { enfermagem. Pesquisa } \\
\text { descritiva de abordagem } \\
\text { qualitativa. }\end{array}$ & $\begin{array}{l}\text { Como resultados } \\
\text { evidenciou-se que a equipe } \\
\text { de enfermagem conseguiu } \\
\text { atender as mulheres que } \\
\text { chegaram ao hospital. } \\
\text { Porém ainda com falhas. }\end{array}$ \\
\hline $\begin{array}{l}\text { Percepções de enfermeiros } \\
\text { da atenção primária no } \\
\text { atendimento às mulheres } \\
\text { vítimas de violência sexual(11) }\end{array}$ & $\begin{array}{c}\text { Analisar a percepção } \\
\text { dos enfermeiros sobre } \\
\text { o atendimento às } \\
\text { mulheres vítimas de } \\
\text { violência sexual na } \\
\text { atenção primária. Estudo } \\
\text { de natureza descritivo- } \\
\text { exploratório com } \\
\text { abordagem qualitativa. }\end{array}$ & $\begin{array}{c}\text { A empatia foi um } \\
\text { sentimento presente } \\
\text { nos enfermeiros, bem } \\
\text { como a frustação; a } \\
\text { falta de conhecimento } \\
\text { específico sobre a temática. } \\
\text { Dificuldade na identificação } \\
\text { dos casos de violência } \\
\text { sexual que podem resultar } \\
\text { na subnotificação. }\end{array}$ \\
\hline $\begin{array}{c}\text { A enfermeira desvelando o } \\
\text { significado do atendimento } \\
\text { às vítimas de violência sexual } \\
\text { expresso pelos profissionais } \\
\text { de saúde(12) }\end{array}$ & $\begin{array}{l}\text { Desvelar o significado } \\
\text { da vivência no cuidar } \\
\text { de vítimas de violência } \\
\text { sexual. Pesquisa } \\
\text { qualitativa de abordagem } \\
\text { fenomenológica. }\end{array}$ & $\begin{array}{l}\text { Profissionais retrataram } \\
\text { que se sentem impotentes, } \\
\text { ficam com psicológico } \\
\text { abalado. Se sentem inúteis, } \\
\text { impotentes o que gera uma } \\
\text { angustia e grande estresse. }\end{array}$ \\
\hline $\begin{array}{l}\text { Percepções dos profissionais } \\
\text { de saúde de Angola sobre a } \\
\text { violência contra a mulher na } \\
\text { relação conjugal(13) }\end{array}$ & $\begin{array}{l}\text { Identificar as percepções } \\
\text { dos profissionais de } \\
\text { saúde de Angola sobre } \\
\text { violência contra a mulher } \\
\text { na relação conjugal. } \\
\text { Estudo exploratório de } \\
\text { cunho qualitativo }\end{array}$ & $\begin{array}{l}\text { Os resultados desta } \\
\text { pesquisa revelam que os } \\
\text { profissionais de saúde } \\
\text { não estão capacitados } \\
\text { para lidarem com os casos } \\
\text { de mulheres vítimas de } \\
\text { violência conjugal. }\end{array}$ \\
\hline $\begin{array}{c}\text { O acolhimento do profissional } \\
\text { da saúde no atendimento à } \\
\text { mulher vítima de violência } \\
\text { sexual(14) }\end{array}$ & $\begin{array}{l}\text { Investigar na ficha de } \\
\text { primeiro atendimento à } \\
\text { mulher vítima de violência } \\
\text { sexual a conduta do } \\
\text { profissional de saúde nos } \\
\text { procedimentos. Estudo } \\
\text { descritivo baseado na } \\
\text { análise quantitativa. }\end{array}$ & $\begin{array}{l}\text { Foi observado que } \\
\text { modificações como: } \\
\text { oferecer cursos de } \\
\text { capacitação permanentes } \\
\text { para os profissionais } \\
\text { da saúde que assistem } \\
\text { as mulheres vítimas } \\
\text { de violência sexual, } \\
\text { incluída nos currículos de } \\
\text { graduação. }\end{array}$ \\
\hline
\end{tabular}

e a análise dos textos serviram de base para a revisão sistemática realizada (Quadro 1).

A seleção dos estudos para a avaliação proposta nesta revisão obedeceu à sequência: inicialmente com a pesquisa dos DeCS para a busca nas bases de dados, após essa busca foi realizado um levantamento na LILACS e na BDENF. Exclusão de estudos repetidos e que não se encaixavam no tema, seleção dos que responderam à pergunta norteadora e resumos para realizar a revisão.

Como dificuldade para o estudo, encontramos a baixa quantidade de artigos sobre assunto abordado. Isso trava o desenvolvimento de uma discussão mais profunda sobre o assunto.

\section{RESULTADOS}

A pesquisa realizada por Batistetti et al.(10) é descritiva com uma entrevista categorizada com mulheres que se encaixavam na delimitação do estudo. $\mathrm{O}$ estudo foi categorizado em três tópicos; a percepção da mulher quanto ao atendimento recebido; o atendimento a ela prestado pela equipe de enfermagem; e os sentimentos vivenciados pela mulher decorrentes dos atendimentos. Foi constatado que a maioria das mulheres soube reconhecer quem era o profissional que lhe prestou o primeiro atendimento. $\mathrm{O}$ atendimento prestado foi classificado como bom pela maior parte das entrevistadas. A enfermagem conseguiu atingir a maioria das mulheres no quesito tranquilidade $\mathrm{e}$ calma. Mesmo diante de relatos positivos, ainda ocorreram situações nas quais a mulher não se sentiu bem com o atendimento.

O estudo realizado por Mota e Aguiar(11) é descritivo-exploratório com abordagem qualitativa, por meio de uma entrevista. As questões que serviram de base para a pesquisa foram: atender mulheres vítimas de violência sexual pode despertar algum sentimento?; quais são esses sentimentos?; como é atuar nesse tipo de atendimento? Para isso, foram entrevistados 7 enfermeiros, que responderam ser empatia o sentimento despertado, mas ocorreram sentimentos como frustração, o que pode gerar intolerância e descaso diante da vio- 


\begin{tabular}{|c|c|c|}
\hline $\begin{array}{c}\text { O acolhimento do profissional } \\
\text { da saúde no atendimento à } \\
\text { mulher vítima de violência } \\
\text { sexual(14) }\end{array}$ & $\begin{array}{l}\text { Investigar na ficha de } \\
\text { primeiro atendimento à } \\
\text { mulher vítima de violência } \\
\text { sexual a conduta do } \\
\text { profissional de saúde nos } \\
\text { procedimentos. Estudo } \\
\text { descritivo baseado na } \\
\text { análise quantitativa. }\end{array}$ & $\begin{array}{c}\text { Foi observado que } \\
\text { modificações como: } \\
\text { oferecer cursos de } \\
\text { capacitação permanentes } \\
\text { para os profissionais } \\
\text { da saúde que assistem } \\
\text { as mulheres vítimas } \\
\text { de violência sexual, } \\
\text { incluída nos currículos de } \\
\text { graduação. }\end{array}$ \\
\hline
\end{tabular}

Fonte: Elaborado pelo autor,2020.

lência contra a mulher. Para a questão de como atuar em tal situação, a maioria das respostas mostrou despreparo e insegurança do profissional de saúde.

Corrêa(12), em estudo de pesquisa qualitativa de abordagem fenomenológica, entrevistou 12 profissionais de saúde que trabalham em 3 hospitais. Desses, 4 são enfermeiros. A pergunta para nortear a pesquisa foi sobre a experiência de cuidar de vítimas de violência sexual. Como resultado, retrataram como essa assistência abala o estado psicológico dos profissionais, mostrando que eles se sentiam impotentes, angustiados e inúteis, diante de tais situações.

Em estudo exploratório qualitativo(13) foi realizado entrevista com 13 profissionais, dentre os quais 3 enfermeiros. Como resultado, destaca-se que os profissionais de saúde não estão capacitados para lidar com casos de violência sexual. Foi constatado que ainda faltam profissionais capacitados para atender a essas mulheres violentadas.

Um estudo descritivo realizado por Ayres et al.(14) mostrou que 64\% das mulheres que chegaram a unidade de atendimento relatando abuso sexual recebeu dose de contracepção, $61 \%$ recebeu profilaxia de HIV, $85 \%$ receberam profilaxia contra IST's e $77 \%$ contra hepatite B. Foi mostrado que apesar dos profissionais receberem capacitações ainda é insuficiente, que a disciplina de a temática de violência sexual deveria ser incluída na grade dos cursos de saúde para que haja cada vez mais capacitações para os profissionais.

\section{DISCUSSÃO}

A violência sexual é um assunto conside- rado um problema de saúde pública. Deve ser analisada com um olhar minucioso. Ao longo dos anos, vem crescendo esse número de vítimas. Com isso, a equipe de enfermagem deve ser organizada e capacitada, a fim de conseguir suprir a necessidade de atendimento às vítimas de violência sexual. Em qualquer que seja a fase da vida da mulher, ela deve ser atendida de forma humanizada e empática(5). Os estudos mostraram o despreparo dos profissionais de enfermagem para atender às mulheres vítimas de violência sexual.

A escassez de conhecimento de como a violência sexual pode atingir a vida de uma mulher pode gerar uma frieza na hora do atendimento à vítima. Com isso, a paciente irá ser acolhida em um modelo bem biomédico e mecânico, focando paritariamente no cuidado físico dela. Corrêa(12) cita em seu trabalho como é importante compreender a vivência da mulher: "Compreendo que a vivência sofrida pela mulher desse tipo de abuso exige que os profissionais de saúde dediquem maior atenção ao "ser" mulher, além do aspecto biomédico.”

Um estudo realizado(11) reforçou que a falta de informação por parte da equipe de enfermagem causa mau atendimento biomédico, o que dificulta a criação de vínculo entre paciente e profissional.

Com isso, faz-se necessário pensar a importância do profissional de saúde para o acolhimento de tais pacientes, sendo que é preciso que eles saibam como agir diante de uma situação de violência sexual. O encaminhamento das clientes é de extrema importância, porém é necessário que os enfermeiros(as) estejam preparados para atender a essa mulher, durante a chegada, como ponto de apoio de saúde(15).

A capacitação dos profissionais para o atendimento é de extrema importância. Todos os estudos mostraram que ainda falta uma educação continuada e especializada aos profissionais, por falha do próprio sistema que deveria, além de executar uma seleção minuciosa desses profissionais, oferecer capacitações.

Mota e Aguiar(11) mostraram que a falta de capacitação leva a uma transferência precoce da vítima, antes mesmo da criação de um vínculo para levantamento de informações. Isso pode gerar subnotificação dos casos de violência sexual. Diante dessas informações, faz-se necessário conhecimento maior acerca do assunto.

Nos EUA, há um programa de examinadores de enfermagem de agressão sexual, para evitar os casos de atender essas vítimas sem o auxílio dos profissionais capacitados. Com isso, $\operatorname{Adams}(16)$ realizou uma pesquisa relacionada ao programa, para não ocorrer o atendimento das vítimas por profissionais fora do programa, o que podemos relacionar com o atendimento brasileiro sem uma equipe específica para o atendimento de vítimas de violência sexual.

Corrêa(12) apontou que os profissionais se sentem exaustos e sem preparo psicológico. Isso gera uma sobrecarga dos profissionais, e eles sentem a dor da vítima e sofrem com ela. Faz-se necessária a contratação de profissionais que tenham uma saúde mental adequada ao atendimento de mulheres em situação de vulnerabilidade e vítimas de violência sexual.

Um ponto importante, que é possível ligar-se diretamente com o preparo dos profissionais e foi apontado na pesquisa de Carneiro et al.(17), é de que, quando se trata de violência contra a mulher, é necessário que profissional saiba como agir, assim que a cliente chegar a unidade. É preciso fazer uma interlocução entre à unidade de saúde e os órgãos públicos aos quais as vítimas devem ser encaminhadas, bem como realizar um boletim de ocorrência e acompanhamento psicológico.

Esse contato permite acolher, investigar e conquistar a confiança da paciente, para que ela se sinta confortável para dividir 
com o profissional essa experiência dolorosa e difícil de ser relatada por ela. Esse olhar diferencial é de extrema importância para construir um vínculo entre a equipe $\mathrm{e}$ a vítima. Isso reflete diretamente em como a cliente irá se sentir, durante o atendimento(10).

É necessário que o hospital que atenda vítimas de violência sexual ofereça a seus profissionais cursos e capacitações de como deve ser realizado esse atendimento e, na hora da seleção, priorize profissionais com perfil mais adequado ao setor de atendimento a mulheres em situação de violência. Isso é imprescindível ao acolhimento, assistência técnica, tratamento empático e humanizado.

E apesar dos cursos de capacitaçōes ainda falta preparo dos profissionais quanto ao atendimento das vítimas de violência sexual. Uma solução além de treinamentos seria a adição de tal temática na grade das faculdades de saúde para que os estudantes saiam da graduação com um melhor conhecimento do assunto e que possa oferecer o melhor atendimento a essas vítimas(14).

\section{CONCLUSÃO}

Conclui-se que a violência a mulheres é uma questão de saúde pública e que essas vítimas estão vulneráveis a sintomas psiquiátricos, colocando a enfermagem na linha de frente para detectar esses sintomas ali presentes, uma vez que o enfermeiro presta esse acolhimento na consulta de enfermagem na rede pública.

Apesar de todos os protocolos, preparo teórico, prático e embasamento científico ao longo da formação acadêmica do profissional de saúde, o atendimento de enfermagem ainda é falho. É preciso haver uma equipe multidisciplinar alinhada e capacitada para acolher e dar continuidade ao atendimento humanizado às mulheres vítimas de violência sexual.

Tanto na rede pública quanto na priva$\mathrm{da}$, uma das alternativas para preparar esses profissionais vem sendo a educação continuada, a fim de preparar e padronizar esses atendimentos, em busca de um perfil adequado para ocupar o cargo de atendimento a vítimas de violência sexual.

A enfermagem tem como papel o "cuidar", dando, assim, oportunidade de criação de vínculo entre cliente e profissional, para obter várias informações que vão resultar em tratamento humanizado e menos invasivo possível. É de extrema importância que os profissionais que irão atender a essas vítimas sejam capacitadas para conseguir criar esse vínculo, principalmente em casos de violência ou abuso sexual.

\section{REFERÊNCIAS}

1. Nunes MCA, Lima RFF, Morais NA. Violência sexual contra mulheres: um estudo comparativo entre vitimas adolescentes e adultas. Psicol Cienc Prof [Internet]. 2017 [acesso em 02 jul 2019];37(4):956-69. Disponivel em: http://dx.doi.org/10.1590/1982-3703003652016

2. Facuri CO, Fernandes AMS, Oliveira KD, Andrade TS, Azevedo. Violência sexual: estudo descritivo sobre as vítimas e o atendimento em um serviço universitário de referência no Estado de São Paulo, Brasil. Cad Saúde Pública [Internet]. 2013 [acesso em 17 jul 2019];5(29):889-98. Disponível em: http://dx.doi.org/10.1590/S0102-311X2013000500008

3. Pinto LSS, Oliveira IMP, Pinto ESS, Leite CBC, Melo AN, Deus MCBR. Women's protection public policies: evaluation of health care for victims of sexual violence. Cienc Saúde Coletiva [Internet] 2017 [acesso em 11 jul 2019];22(5):1501-8. Disponível em: http:/dx.doi.org/10.1590/141381232017225.33272016

4. Ministério da Saúde (BR). Secretaria de Atenção à Saúde. Departamento de Ações Programáticas Estratégicas. Prevenção e tratamento dos agravos resultantes da violência sexual contra mulheres e adolescentes: norma técnica [Internet]. $3^{\text {a }}$ ed. atual. e ampl., 1a reimpr. Brasília (DF): MS, 2012 [acesso em 13 dez 2019]. Disponivel em: https:/bvsms.saude.gov.br/bvs/publicacoes/prevencao_agravo_violencia_sexual_mulheres_3ed.pdf

5. Huçulak M, Ferreira MCF, Tchaikovski HLD. Protocolo para o atendimento às pessoas em situação de violência sexual / SAS [Internet]. Secretaria de Estado da Saúde do Paraná (PR). Superintendência de Atenção à Saúde. $2^{\text {a }}$ ed. Curitiba (PR): SESA, 2017 [acesso em 02 nov 2019]. Disponivel em: https:/www.saude.pr.gov.br/sites/default/arquivos_restritos/files/documento/2020-04/protocolo_apsvs_ultimaversao.pdf

6. Higa R, Mondaca ADCA, Reis MJ, Lopes MHBM. Atendimento à mulher vítima de violência sexual: protocolo de assistência de Enfermagem. Rev Esc Enferm USP [Internet]. 2008 [acesso em 05 jul 2019];42(2):377-82. Disponivel em: http://dx.doi.org/10.1590/50080-62342008000200023

7. Ministério da Saúde (BR). Saúde divulga diretrizes para atendimento a vítimas de violência sexual [Internet]. Brasília (DF): MS, 2015 [acesso em 15 set 2019]. Disponivel em: http:/www.saude.gov. br/noticias/svs/20306-saude-divulga-diretrizes-para-atendimento-a-vitimas-de-violencia-sexual

8. Woiski ROS, Rocha DLB. Cuidado de enfermagem à criança vítima de violência sexual atendida em unidade de emergência hospitalar. Esc Anna Nery [Internet]. 2010 [acesso em 23 out 2019];14(1):143-50. Disponivel em: https:/doi.org/10.1590/S1414-81452010000100021

9. Morais SCRV, Monteiro CFS, Rocha SS. O cuidar em enfermagem à mulher vítima de violência sex- ual. Texto Contexto-Enferm [Internet]. 2010 [acesso em 07 ago 2019];19(1):155-60. Disponível em: https:/doi.org/10.1590/S0104-07072010000100018

10. Batistetti LT, Lima MCD, Souza SRRK. The perception of sexual violence's victims about the embracement in a reference hospital in Paraná. Rev Pesq (Univ Fed Estado Rio J, Online) [Internet]. 2020 [acesso em 02 ago 2020];12:169-75. Disponivel em: http:/dx.doi.org/10.9789/2175-5361. rpcfo.v12.7191

11. Mota JA, Aguiar RS. Percepções de enfermeiros da atuação primaria no atendimento as mulheres vítimas de violência sexual. Nursing (São Paulo) [Internet]. 2020 [acesso em 02 ago 2020];23(262):3648-51. Disponivel em: http://www.revistanursing.com.br/revistas/262/pg31.pdf 12. Corrêa MEC. A enfermeira desvelando o significado do atendimento às vitimas de violência sexual expresso pelos profissionais de saúde [tese]. Curitiba: Universidade Federal do Paraná; 2008 [acesso em 22 nov 2019]. Disponivel em: https:/acervodigital.ufpr.br/bitstream/handle/1884/14702/DISSERTACAO_MESTRADO_MARIA_EDUARDA_CAVADINHA_CORREA.pdf?sequence $=1$ \&isAllowed $=y$

13. Nascimento EFGA, Ribeiro AP, Souza ER. Perceptions and practices of Angolan health care professionals concerning intimate partner violence against wornen. Cad Saúde Pública [Internet]. 2014 [acesso em 13 nov 2019];30(6):1229-38. Disponivel em: https://doi.org/10.1590/0102$311 \times 00103613$

14. Ayres $L F$, Amorim $W M$, Silva $L R$. $O$ acolhimento do profissional da saúde no atendimento à mulher vítima de violência sexual. Rev Enferm UFPE On Line [Internet]. 2009 [acesso em 20 ago 2020];3(4):962-71. Disponivel em: https://doi.org/10.5205/reuol.581-3802-1-RV.0304200922 15. Silva NNF, Leal SMC, Trentin D, Vargas MAO, Vargas CP, Vieira LB. Atuação dos enfermeiros da atenção básica a mulheres em situação de violência. Enferm Foco [Internet]. 2017 [acesso em 18 nov 2019];8(3):70-4. Disponivel em: file://C:/Users/User/AppData/Local/Temp/1290-5213-1-PB. pdf

16. Adams $P$. Hulton $L$. The sexual assault nurse examiner's interactions in sexual assault response team: a systematic review. Adv Emerg Nurs J [Internet]. 2016 [acesso em 02 jul 2019];38(3):21327. Disponivel em: 10.1097/TME.0000000000000112

17. Carneiro JB, Gomes NP, Estrela FM, Paixão GPN, Romano CMC, Mota RS. Unveiling the strategies used by women for confronting marital violence. Texto Contexto-Enferm [Internet]. 2020 [acesso em 11 ago 2020];29:e20180396. Disponivel em: http:/dx.doi.org/10.1590/1980-265xtce-2018-0396 